\title{
An Update on Regenerative Medicine Clinical Trials in Erectile Dysfunction: Have We Made Any Progress?
}

\author{
Mohit Khera $^{a, *}$, Trinity Bivalacqua ${ }^{b}$, Irwin Goldstein $^{c}$, Maarten Albersen $^{\text {d,e }}$ \\ ${ }^{a}$ Baylor College of Medicine, Houston, TX, USA; ${ }^{\mathrm{b}}$ The James Buchanan Brady Urological Institute, Johns Hopkins Hospital, Baltimore, MD, USA; ${ }^{\mathrm{c}}$ Sexual \\ Medicine, Alvarado Hospital, San Diego, CA, USA; d Laboratory of Experimental Urology, Department of Development and Regeneration, Leuven University, \\ Leuven, Belgium; ' Department of Urology, University Hospitals Leuven, Leuven, Belgium
}

\section{Introduction}

In a 1998 study published at the time of marketing of the first available phosphodiesterase type 5 inhibitor sildenafil, the highest rank measure of treatment success in terms of importance for erectile dysfunction (ED) patients was "cure" [1]. Although we have several options for improving ED in our armamentarium, in the $20 \mathrm{yr}$ since the first effective oral therapy, we have not been able to offer ED patients what they desire most. A cure for ED that results in spontaneous unassisted intercourse requires research into novel disease-modifying regenerative treatment methods such as stem cell therapies.

To date, results have been published from four singlearm trials conducted in human patients. Bahk et al [2] tested administration of umbilical cord stem cells in seven diabetic patients awaiting a penile implant, of whom six experienced an improvement in erectile function. In 2016, Haahr et al [3] treated 17 patients after radical prostatectomy with an intracavernous injection of regenerative cells derived from adipose tissue. At 6 mo after injection, eight men (47\%) had regained their erectile function and were able to accomplish sexual intercourse. Interestingly, no effects were seen at 1 mo after injection in this study, which argues against a placebo effect and in favor of induction/stimulation of regenerative processes. Yiou and colleagues [4] used intracavernous injection of bone marrow mononuclear cells for patients suffering from ED after radical prostatectomy in the INSTIN phase 1-2 trial. The authors concluded that erectile function was significantly improved after 6 mo for patients given $1 \times 10^{9}$ cells, with erectile function domain scores on the International Index of Erectile Function (IIEF-EF) of $18 \pm 8.3$ versus $3.7 \pm 4.1$ at baseline. No serious side effects occurred after mean follow-up of 62.1 mo for the first 12 patients, and no increase in the risk of prostate cancer recurrence was identified. Al Demour et al [5] carried out a single-arm phase 1 trial published in 2018 in which four diabetic ED patients experienced no side effects and had an IIEF-EF improvement of approximately 12 points after intracavernous injection of autologous bone marrow derived mesenchymal stem cells.

While these all are phase 1 open-label noncontrolled trails, in all instances the authors have made claims regarding efficacy. This is troublesome, as a large proportion of these patients are prone to experiencing spontaneous ED improvement (inclusion relatively shortly after nerve-sparing radical prostatectomy) and the placebo effect can be very prominent in ED research. To illustrate this second point, Carvalho De Araujo and coworkers [6] designed a prospective, controlled, single-blinded, parallel-group study with 123 ED patients, all of whom received placebo treatment. Even though patients were aware of this, they experienced an average increase in IIEF-EF score of 4 points. The important lesson to learn from these data is that ED patients under active treatment in a study setting are likely to experience an improvement in their IIEF scores. Hence, single-arm studies and open-label noncontrolled trails should be regarded as hypothesis-generating and should not be included in an evidence review for making practice recommendations or guidelines. In all the studies that have been reported, side effects have been limited to minor side effects such as mild pain or bruising at the site of harvesting

* Corresponding author. 7200 Cambridge Street 10th floor, Houston, Texas 77030. Tel.: +1 713 7986593; Fax: +1 7137985553 .

E-mail address: mkhera@bcm.edu (M. Khera). 